Sir,

\section{Charles Bonnet Syndrome following enucleation}

Complex visual hallucinations in the presence of a clear mental state, have received increased recognition since Charles Bonnet's original depiction of visual hallucinations secondary to mature cataracts in his grandfather, Charles Lullen in $1769 .{ }^{1}$ Over the past few years it has become clear that the previously reported incidence of up to $15 \%^{2}$ is probably a gross underestimate for the condition.

We present a case of complex visual hallucinations following unilateral enucleation of a patient with excellent vision in the remaining eye, sharing many features of the Charles Bonnet Syndrome (CBS). We believe this to be the first reported case of CBS in an enucleated eye since Uhthoff's reference to a similar case in $1899 .^{3}$

\section{Case report}

A 65-year-old married and articulate middle-class artist, presented asymptomatically as a tertiary referral with a presumed diagnosis of choroidal melanoma in his right eye. Initial examination revealed visual acuity of $6 / 12$ right and 6/6 in the left unaided. Although the anterior segments were normal, fundal examination showed a lesion $12 \mathrm{~mm}$ in largest ultrasound base diameter, and $7 \mathrm{~mm}$ in height, measuring $7 \mathrm{~mm}$ from the optic disc in the right eye. The lesion extended anteriorly to the ciliary body in the supero-temporal quadrant. Surface lipofuscin, serous retinal detachment, and moderate pigmentation of the lesion were noted. Following counselling of the patient, an uncomplicated enucleation with a hydroxyapatite implant was preformed. The patient was discharged home 2 days later.

After 9 months, on routine review of his cosmetic prosthesis, he described complex vivid visual hallucinations, which had started approximately 3 months after surgery, and which appeared several times daily. The images appeared spontaneously in the presence of a clear sensorium, and were described as rural landscapes striking for their detail and richness of colour, predominantly yellow and green. The gentleman retained good insight, knowing these images were not real, and though not distressed, was frustrated by his complete inability to capture them on canvas. He further described sudden and complete cessation of these images by closing his enucleated eye. The hallucinations returned once the eye was opened. He was otherwise fit and well and had taken no new medication.

\section{Comment}

Typically, CBS hallucinations are described as a sudden focused image, sharp and immobile, often that of a person, a face, a flower, or a regular pattern. The visual hallucinations usually occur when the eyes are open and vanish spontaneously. ${ }^{4}$ In fact, eye closure has been documented to both extinguish the hallucinations and on other occasions bring on the visual hallucinations. ${ }^{5}$ Symptoms of CBS often occur in the context of sudden and unexpected reduction in visual function, ${ }^{6}$ and may occur after a latent period of days to weeks after visual impairment.

Our case highlights many of these features, and although visual impairment is not bilateral, the afferent sensory stimulus has declined to levels allowing suppressed stimuli to become conscious, manifesting as real, vivid complex hallucinations. Although the mechanism of these CBS symptoms is poorly understood, such phenomena may be conceptualised as phantom vision (similar to the phantom limb syndrome $e^{7}$, or related to the Perceptual Release Theory of West. ${ }^{8}$

The deprivation of normal afferent input to the visual cortex may result in the cortex responding with impulsive independent activity and resultant complex imagery. Increasing the stimulus level to the previous normal state results in abolition of hallucinations, supporting the phantom vision theory. ${ }^{9}$ This may explain the abolition of CBS symptoms on eye closure in our patient, with secondary normalisation of sensory input in the dark.

However, the perceptual release theory, suggests that a normal sensory input results in the brain excluding irrelevant impulses from the conscious perception of images. This occurs as a result of an intrinsic active censorship mechanism the brain possesses. With a reduction in sensory input, as occurs in visual loss, the threshold to suppress irrelevant images cannot be achieved and previously subconscious perceptions emerge into consciousness, resulting in a visual hallucination.

CBS is an important symptom, often left unnoticed with the patients in fear of being branded insane. It is therefore important to understand that visual hallucinations can occur in eyes with previously excellent vision following sudden profound insults to visual function such as enucleation.

\section{References}

1 Bonnet C. Essai analytique sur les facultes de l'ame, 2nd ed. Philibert: Copenhagen and Geneva, 1769, pp 176-178.

2 Menon GJ, Rahman I, Menon SJ, Dutton GN. Complex visual hallucinations in the visually impaired: the Charles Bonnet Syndrome. Surv Ophthalmol 2003; 48: 58-72 (Major Review). 
3 Duke-Elder S, Scott GI. Disorders of perception: visual hallucinations. In: Duke-Elder S (ed) System of Ophthalmology, Vol. XII. Henry Kimpton: London, 1971, pp 562-569.

4 Schultz G, Needham W, Taylor R, Shindell S, Melzack R. Properties of complex visual hallucinations associated with deficits in vision. Perception 1996; 25: 715-726.

5 Teunisse RJ, Cruysberg JR, Hoefnagels WH, Kuin Y, Verbeek AL, Zitman FG. Visual hallucinations in psychologically normal people: Charles Bonnet's Syndrome. Lancet 1996; 347: 794-797.

6 Holroyd S, Rabins PV, Finkelstein D, Nicholson MC, Chase GA, Wisniewski SC. Visual hallucinations in patients with macular degeneration. Am J Psychiatry 1992; 149: 1701-1706.

7 Cohn R. Phantom vision. Arch Neurol 1971; 25: 468-471.

8 Asaad G, Shapiro B. Hallucinations: theoretical and clinical overview. Am J Psychiatry 1986; 143: 1088-1097.

9 Bartlett JEA. A case of organised visual hallucinations in an old man with cataract, and their relationship to the phenomena of the phantom limb. Brain 1951; 74: 363-373.

J Ross ${ }^{1}$ and I Rahman ${ }^{2}$

${ }^{1}$ St Pauls Eye Unit, Liverpool, UK

${ }^{2}$ Manchester Royal Eye Hospital

Nelson Street Manchester

M13 9WL, UK

Correspondence: I Rahman

Tel: + 441612761234

Fax: + 441612726618

E-mail: imran1973@tiscali.co.uk

Conflict of interest: None.

Financial interest: None.

Eye (2005) 19, 811-812. doi:10.1038/sj.eye.6701647; published online 17 September 2004

Sir,

Light-near dissociation of pupil reactions as a presenting feature of von Hippel-Lindau disease

Bilateral light-near dissociation of the pupil reactions is typically caused by rostral midbrain lesions, which interrupt the retinotectal input to the Edinger-Westphal nuclei, but not the more ventral accommodative input. ${ }^{1}$ We observed light-near dissociation of the pupil reactions as a presenting feature in a patient with a cerebellar haemangioblastoma, and who was subsequently diagnosed with von Hippel-Lindau disease.

A 38-year-old heating engineer presented to us with a 10 -week history of blurred distance vision, headaches, impaired balance, vertigo, and fatigue. The deterioration in distance vision was bilateral and not associated with any diplopia.

His corrected acuities were $6 / 6$ in each eye, but he had large dilated pupils, which reacted poorly to light but well to near targets. There was no segmental paralysis or vermiform movements evident on slit-lamp examination. Interestingly, his saccadic and pursuit eye movements were also normal, with no evidence of eyelid retraction or convergence retraction nystagmus on upgaze.

Dilated fundoscopy revealed multiple retinal haemangioblastomas, and a brain MRI demonstrated a large cystic lesion within the cerebellum. This cystic mass contained a superficial nodule which was enhanced following intravenous gadolinium administration, a feature characteristic of haemangioblastomas. ${ }^{2}$ The mass was surrounded by oedema and was causing distortion of the IV ventricle, dorsal pons, and midbrain. Further investigations demonstrated a left renal mass and a mutation in the von Hippel-Lindau (VHL) gene.

Von Hippel-Lindau disease is an autosomal dominant disorder caused by mutations in a tumour suppressor gene on chromosome 3. Central nervous system (CNS) and retinal haemangioblastomas are the most frequent features of von Hippel-Lindau disease, with CNS haemangioblastoma being present in up to $80 \%$ of patients. ${ }^{3} \mathrm{CNS}$ haemangioblastomas are slow-growing and can reach large sizes in the cerebellum before becoming apparent clinically; light-near dissociated pupil reactions have not, however, been described previously as a presenting feature. In the months following surgical removal of our patient's cerebellar tumour, the light-near dissocation has been seen to become less apparent. We therefore believe the oedema surrounding the tumour, and associated compression of the dorsal midbrain, was the cause of the abnormal pupil reactions. He is currently under follow-up with repeated imaging.

\section{Acknowledgements}

No grants or sponsoring organizations have been involved in the work for this submission. The authors have no proprietary or commercial interests related to the manuscript. It has not been presented previously.

\section{References}

1 Thompson HS. Light-near dissociation of the pupil. Ophthalmologica 1984; 189: 21-23.

2 Torreggiani WC, Keogh C, Al-Ismail K, Munk PL, Nicolaou S. Von Hippel-Lindau disease: a radiological essay. Clin Radiol 2002; 57: 670-680. 